\title{
Surface activity of surfactant spiked with vitamin A
}

\author{
Vadim Bronshtein \\ Vugranam Venkatesh \\ Jas Aulakh \\ Philippe Chessex \\ Division of Neonatology, Children's \\ and Women's Health Centre \\ of British Columbia, University \\ of British Columbia, Vancouver, BC \\ Canada
}

Background: Intramuscular injections of vitamin A decrease the risk of broncho-pulmonary dysplasia. Admixture of vitamin A with surfactant as a lipophilic vehicle might be a less invasive modality.

Aim: Test physical properties of surfactant + vitamin A.

Methods: Miscibility and surface activity were tested in surfactant supplemented with retinylacetate, -palmitate, 13-cis-, or all-trans-retinoic acid.

Results: Retinol acetate $(5000 \mathrm{IU} / \mathrm{mL})$ demonstrated miscibility with surfactant when premixing with ethanol. Its surface activity was $40 \%$ lower compared to surfactant alone.

Conclusion: These findings warrant preclinical studies to test whether administration of vitamin A in subjects requiring surfactant is associated with beneficial functional properties.

Keywords: drug delivery, vitamin A, surfactant, surface tension

\section{Introduction}

Broncho-pulmonary dysplasia (BPD) is characterized by decreased alveolarization, a localized inflammatory response, pulmonary infections, and impaired pulmonary vascular growth (D'Angio and Maniscalco 2004; Stenmark et al 2005). BPD is an important cause of death and long-term disability among extremely low birth weight (ELBW) infants (Smith et al 2004). Conventional treatments for BPD include various modalities of ventilation, high dose systemic corticosteroids, diuretics, antiinflammatory agents, antiinfective agents, and antioxidants (D'Angio and Maniscalco 2004) such as vitamin A (Ambalavanan et al 2004).

Vitamin A is the generic name for a group of fat-soluble compounds which have the biological activity of the primary alcohol, retinol. In vitro and in physiologic animal models, it has been demonstrated that vitamin $\mathrm{A}$ is involved in the regulation and promotion of growth and differentiation of many cells (Ozer et al 2005). In the lung it promotes alveolar repair, protects against hyperoxia mediated cell-cycle arrest, stimulates surfactant synthesis, and improves pulmonary vascularization (Maden et al 2004; Snyder et al 2005). Since retinol accretion occurs during late gestation, premature infants are more prone to vitamin A deficiency (Hustead et al 1984; Shenai et al 1985).

Evidence from randomized trials supports parenteral vitamin A supplementation with reported reduction in death or oxygen requirement (Darlow and Graham 2002) in premature infants ventilated for respiratory distress syndrome (RDS). However, Vitamin A supplementation in ELBW infants is not routinely practiced, perhaps, because the current mode of administration through multiple intramuscular injections is considered invasive (Ambalavanan et al 2004), which accounts for the search for alternate modalities of administration of this lipophilic antioxidant vitamin.

Vitamin A administration is challenging as it may have unwanted effects like increased intracranial pressure, liver toxicity, or drug interactions at high doses (Francisko et al 1993; Ambalavan et al 2003). Oral supplementation with vitamin A may not be efficacious (Wardle et al 2001). Intravenous administration of vitamin A in total 
parenteral nutrition is not satisfactory because of photo degradation (Allwood and Martin 2000) and significant adsorption to the tubing (Haas et al 2002). Contrary to the antioxidant vitamins $\mathrm{C}$ and $\mathrm{E}$ that increase in lungs with infused concentrations, pulmonary levels of vitamin A remain undetectable (Lavoie et al 2007). Vitamin A compounds administered into the lungs via the upper airway have been shown to be biologically active in humans (Biesalski et al 1999; Kohlhaufl et al 2002), which suggests that this may be a route of administration worth investigating in premature infants.

A majority of premature ELBW infants with RDS receive exogenous surfactant which has surface tension properties that contribute to improve the ventilation perfusion ratio of the immature lungs. The high phospholipid and apoprotein content of surfactant account for its lipophilic properties. It was deemed of therapeutic interest to test whether vitamin A can be co-administered with surfactant as a lipophilic vehicle to provide an alternate, less invasive, approach to providing this antioxidant vitamin in premature infants who require surfactant replacement therapy. The objective of this study was to determine in vitro the effect of the admixture of vitamin A with a clinically relevant surfactant preparation on miscibility and surface tension properties.

\section{Methods}

We tested the miscibility/solubility and surface activity of bovine surfactant (BLES Biochemicals Inc., London, On, Canada) \pm alcohol (100\% denatured, Sandoz, Boucherville, QC, Canada) \pm emulsifiers (tween 80 : polyoxyethylenesorbitan monooleate from Sigma-Aldrich, Canada; tyloxapol: 4-(1,1,3,3-tetramethylbutyl) phenolpolymer with formaldehyde and oxirane from Sigma-Aldrich, Canada) supplemented with 5000 or $50000 \mathrm{IU} / \mathrm{mL}$ of the following vitamin A derivatives: -retinly acetate, -retinyl palmitate, -13-cis retinoic acid, -all-trans retinoic acid (ATRA) from Sigma-Aldrich, Canada. The bovine surfactant was provided graciously for the study by BLES Biochemicals Inc.

To prepare the "surfactant + vitamin A" solution, 5000 or 50000 IU of vitamin A derivatives were dissolved directly and vortexed into $1 \mathrm{~mL}$ BLES. To prepare the "surfactant + vitamin A + alcohol" preparation, a stock solution of 50000 $\mathrm{IU} / \mathrm{mL}$ vitamin A dissolved in alcohol was vortexed with $1 \mathrm{~mL}$ BLES. The $5000 \mathrm{IU} / \mathrm{mL}$ vitamin A solution was obtained by dilution of the $50000 \mathrm{IU} / \mathrm{mL}$ before mixing with $1 \mathrm{~mL}$ BLES. The "surfactant + vitamin A + emulsifiers" preparation was the same as above, apart from using tyloxapol (25 mg) or tween 80 instead of alcohol. Tyloxapol is a strong detergent that contributes to the dispersion of dipalmitoyl phosphatidylcholine in the artifical lung surfactant Exosurf (Burroughs Welcome); whereas tween 80 is a common emulsifier used in laboratories to solubilize hydroand lipophilic compounds.

Miscibility/solubility was determined at room temperature and at $37^{\circ} \mathrm{C}$ immediately upon mixing the preparation and $24 \mathrm{~h}$ thereafter by testing visually for macroscopic appearance and microscopically using light and fluorescence for precipitation (Wong et al 2006). Miscibility relates to two solutions. Solubility relates to a solution and solids. Macroscopic evaluation (Eggert et al 1982) was performed in a plastic test tube after vigorous agitation prior to visual inspection to determine the presence of opalescence, separation of solutions, gross precipitation or crystallization using a strong light and a dark background. Preparations not showing the above physical signs were considered macroscopically miscible. Using a light microscope at standard magnification (10 and $100 \times$ ), evidence of precipitation and crystallization was sought in all preparations, while separation of solutions and/ or denaturation of BLES were observed using a fluorescent microscope. Three determinations were performed for each preparation. The technique was validated by verifying that the limit of precipitation detected microscopically did not vary over multiple samples of the same vitamin A solution.

Surface activity was measured for the vitamin A derivatives exhibiting miscibility in surfactant. Immediately after mixing the "surfactant + vitamin A + ethanol" preparation, surface activity was measured using a pulsating bubble surfactometer (PBS, General Transco Inc., Largo, FL, USA) as described by Enhorning (1977). The mixture of $138 \mu \mathrm{L}$ of the preparation diluted in $1362 \mu \mathrm{L}$ of diluent $(240 \mu \mathrm{L}$ absolute ethanol in $260 \mu \mathrm{L}$ purified water) was kept at $37^{\circ} \mathrm{C}$ for 90 minutes prior to determination of surface tension. The preparation was then loaded and allowed to equilibrate for three minutes in the small volume chamber of the PBS, which communicates with ambient air through a chimney. The motion of a piston of the PBS produces a negative pressure in the chamber until a bubble is formed at the air-liquid interface in the chimney. As the bubble is subjected to 20 pulsations per minute for $2 \frac{1}{2}$ minutes, the surface tension is recorded. Under optimal conditions a surfactant will induce, after a few pulsations, the surface tension to diminish to zero during expiration. During each pulsation the bubble formed at the surface of the air/surfactant interface will oscillate between a maximal surface tension when the bubble has the smallest size at baseline, and a minimal surface tension with the larger bubble size (Enhorning 2001). Three runs were performed per sample. A BLES reference sample was run as control and the 
synthetic surfactant Exosurf was run as a further source of comparison. Standard descriptive statistics are used.

\section{Results}

Partial miscibility/solubility with BLES was achieved for retinyl palmitate, ATRA, and 13-cis retinoic acid (Table 1). Retinyl acetate demonstrated better miscibility in vitro. The miscibility of surfactant + retinyl acetate was improved by premixing vitamin A with ethanol. Miscibility/solubility deteriorated after $24 \mathrm{~h}$ storage at room temperature. Overall, miscibility was marginally better with alcohol than with emulsifiers (tween 80 and tyloxapol). At $37^{\circ} \mathrm{C}$ miscibility/ solubility was not improved.

Surface activity was measured to test the effect of vitamin A supplementation on physical properties of surfactant. As shown is Figure 1, BLES alone was associated after 25 pulsations with a minimum surface tension of 0 , in 2 out of 3 runs (mean $\pm \mathrm{SD}: 2 \pm 2 \mathrm{nN} / \mathrm{m}$ ). Under the same conditions the artificial surfactant Exosurf alone exhibited an average minimum surface tension of $29 \pm 1 \mathrm{nN} / \mathrm{m}$. After 25 pulsations the average minimum surface tension of "BLES + retinyl acetate + alcohol" preparation was $13 \pm 4 \mathrm{nN} / \mathrm{m}$ for the $5000 \mathrm{IU} / \mathrm{mL}$ preparation (Figure 2) and $15 \pm 1 \mathrm{nN} / \mathrm{m}$ for the $50000 \mathrm{IU} / \mathrm{mL}$ preparation.

\section{Discussion}

The intratracheal route of administration is widely used for drugs like epinephrine, atropine sulfate, lidocaine hydrochloride, or naloxone hydrochloride in emergency situations. This route is also used for the delivery of steroids as well as $\beta-2$ agonist in asthma and antibiotics in cystic fibrosis. The potential advantage of administering metabolites of vitamin $\mathrm{A}$ in the lungs is to achieve local bioavailability with decreased systemic side effects.

Inhaled forms of retinyl palmitate have been used successfully in humans for treatment of respiratory epithelium metaplasia (Kohlhaufl et al 2002), with improved blood levels of vitamin A as well as retinol binding protein (Biesalski et al 1999). The biologically active metabolite 13-cis retinoic acid was shown to be effective in inhaled form for the prevention of lung cancer in animal studies, without causing liver toxicity (Dahl et al 2000). An inhaled aerosolized preparation of ATRA, which is the end product of vitamin A metabolism that activates receptors, has been used in animals for targeted pulmonary delivery (Brooks et al 2000). In addition, retinyl acetate is a metabolically inert retinyl ester, which we speculate would lead to elevated cellular concentrations of vitamin A, without affecting metabolic regulatory steps. However, to our

Table I Miscibility/solubility of vitamin A derivatives in bovine surfactant

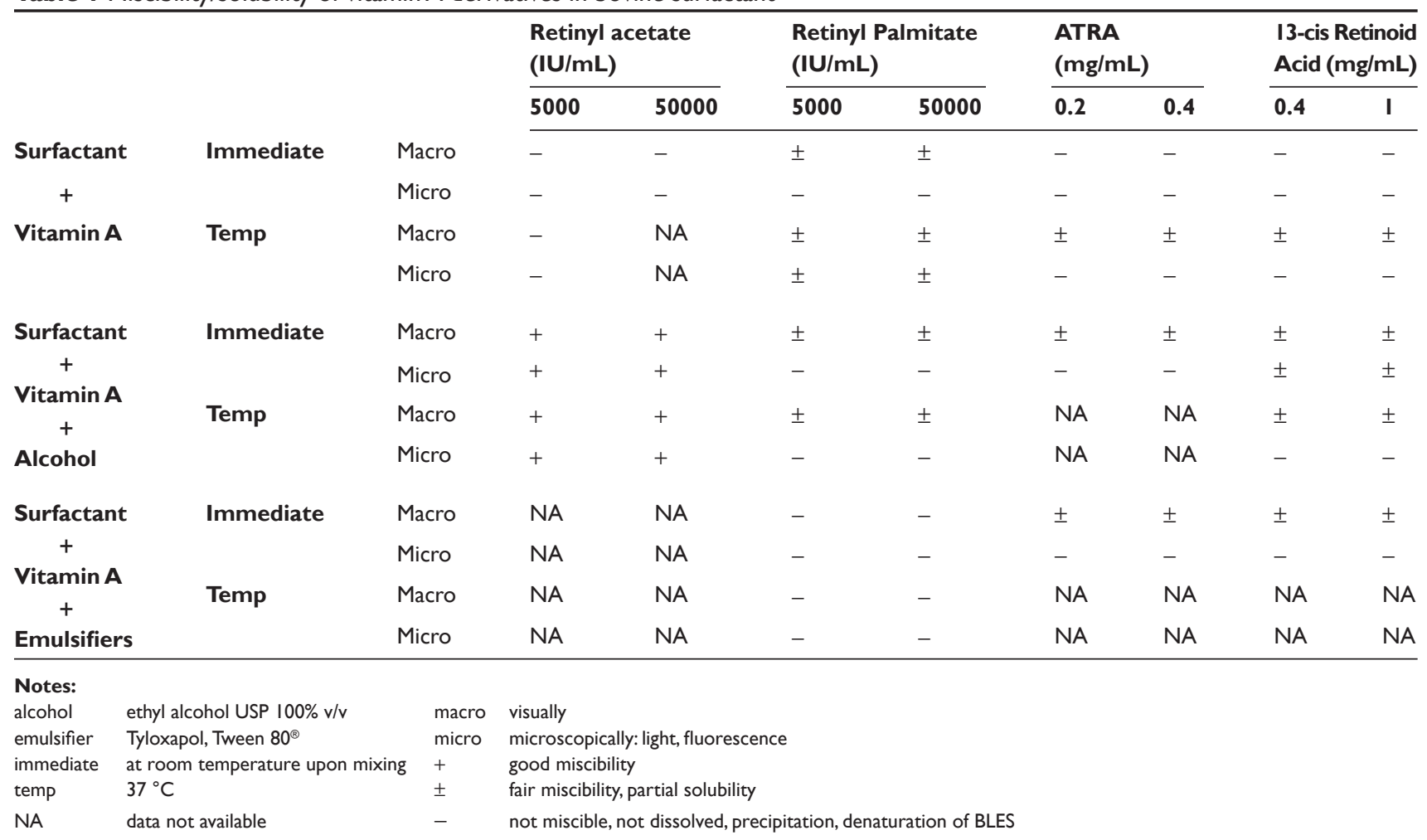




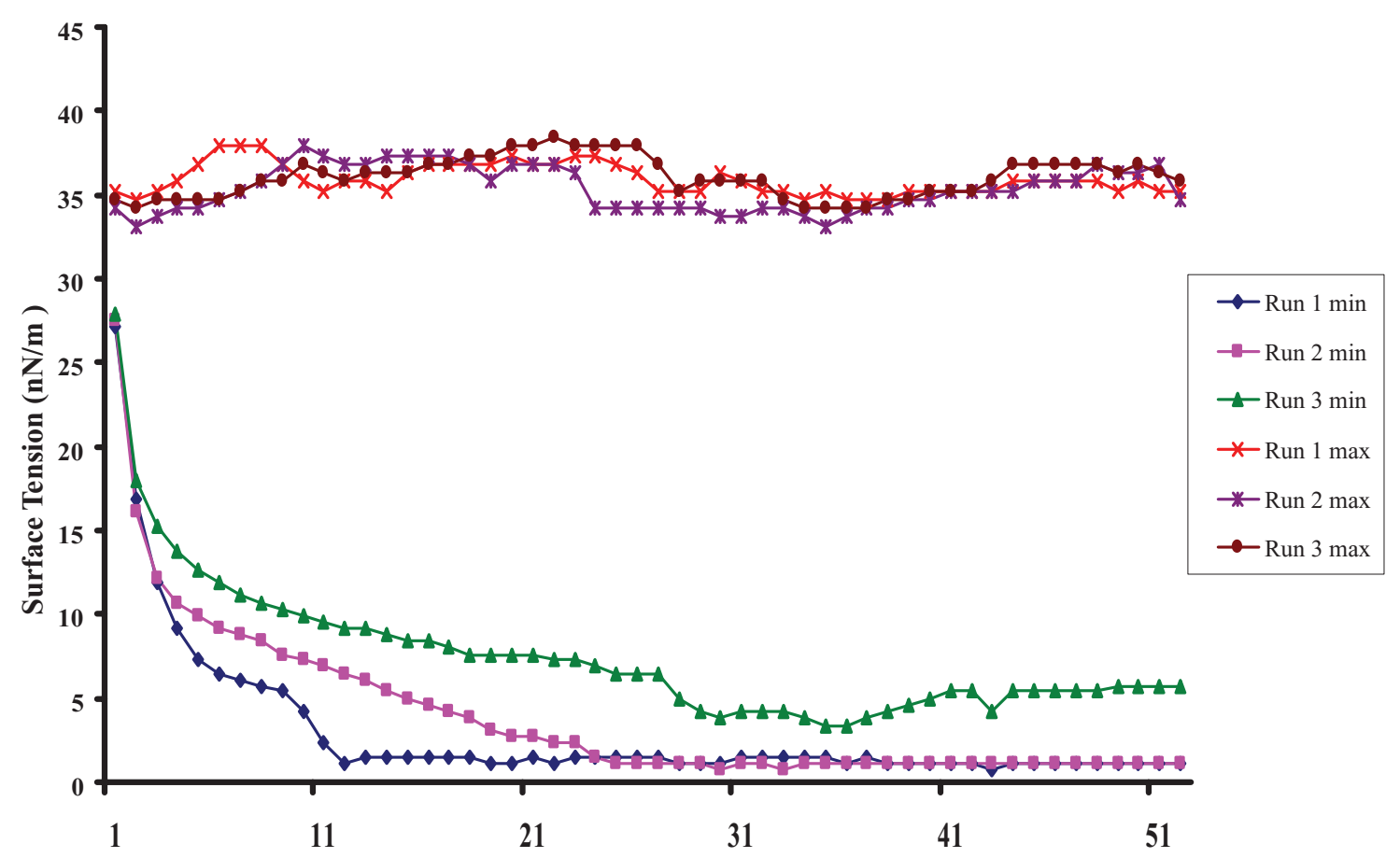

Pulsation Number

Figure I Surface tension properties of surfactant (BLES $\left.{ }^{\circledast}\right)$. Maximum $(\max )$ and minimum $(\mathrm{min})$ surface tension $(\mathrm{nN} / \mathrm{m})$ as determined by pulsating bubble meter in three runs as the bubble formed at the surface of the air/surfactant interface is subject to 20 pulsations per minute for $2 \frac{1}{2}$ minutes. After 25 pulsations the surface tension reaches a minimum of $2 \pm 2 \mathrm{nN} / \mathrm{m}$.

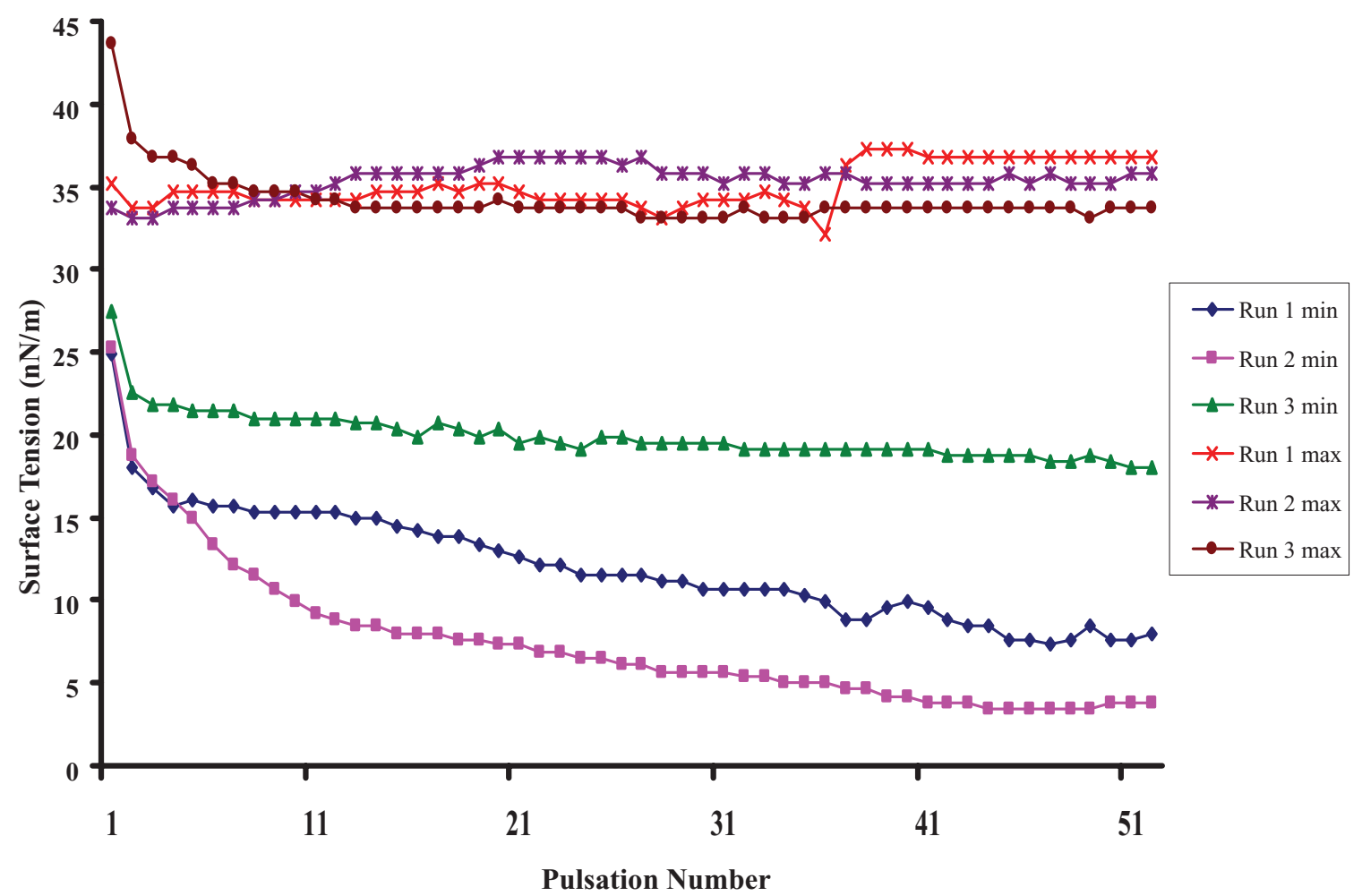

Figure 2 Surface tension properties of the mixture surfactant + retinly acetate + alcohol. Maximum (max) and minimum (min) surface tension ( $\mathrm{nN} / \mathrm{m}$ ) as determined by pulsating bubble meter in three runs as the bubble formed at the surface of the air /surfactant admixture interface is subject to 20 pulsations per minute for $21 / 2$ minutes. After 25 pulsations the surface tension reaches a minimum of $13 \pm 4 \mathrm{nN} / \mathrm{m}$. 
knowledge its administration via the lungs has not been used in humans.

This is the first study evaluating the use of exogenous surfactant as a vehicle for intra-tracheal administration of vitamin A. The results indicate that the retinyl acetate admixture was associated with the better miscibility/solubility in surfactant. Our surface activity values for both BLES and Exosurf surfactants are similar to those previously reported for commercial surfactants from bovine lung extracts or synthetic surfactants (Bernhard et al 2000). We found that the surface activity of the retinyl acetate admixture was better than artificial surfactant, but did not achieve values measured with bovine surfactant alone. However, the significance of these values needs to be considered with caution as physical properties are not a reliable predictor of clinical outcome. The artificial surfactant Exosurf was shown to be associated with beneficial effects on survival of preterm infants with RDS (McMillan et al 1995) in spite of poor in vitro surface tension properties (Bernhard et al 2000). Although the admixture containing 13-cis retinoic acid exhibited surface tension properties that were similar to bovine surfactant alone, its partial miscibility/solubility would be a problem in clinical practice.

The potential advantage of providing vitamin A with surfactant is that babies would receive the antioxidant locally when the oxidant stress is potentially the greatest, as opposed to giving vitamin A intramuscularly (Tyson et al 1999; Darlow and Graham 2002) later when the oxidant damage has already caused structural damage to the pulmonary basement membrane within epithelial cells (D'Angio and Maniscalco 2004). The drawback to this approach is that it provides a limited dose of vitamin A to patients who would receive at the most between 1 to 3 intra-tracheal administrations of surfactant. For this reason we tested a 10 -fold greater concentration of retinyl acetate (50,000 IU), which exhibited a surface tension comparable to the preparation with $5000 \mathrm{IU}(15 \pm 1$ vs $13 \pm 4 \mathrm{nN} / \mathrm{m})$. In preliminaries, the bioavailability of retinyl acetate provided intratracheally with surfactant was documented in animals that showed increased hepatic levels of retinol (Bronstein et al 2005). It remains to be tested whether surfactant spiked with vitamin A will have a greater effect on pulmonary levels than intravenous supplementation (Lavoie et al 2007) and if this is associated with changes in lung function.

Providing vitamin A with surfactant as a lipophilic vehicle may be a less-invasive mode of delivery than by intramuscular injections in subjects requiring surfactant administration. Our findings warrant further preclinical studies to test the biochemical, pharmacological, histological and clinical responses to this noninvasive mode of coadministration of vitamin A.

\section{Disclosure}

The authors report no conflicts of interest in this work.

\section{References}

Allwood MC, Martin HJ. 2000. The photodegradation of vitamins A and E in parenteral nutrition mixtures during infusion. Clin Nutr, 19:339-42.

Ambalavan N, Wu TJ, Tyson JE, et al. 2003. A comparison of three vitamin A dosing regimens in extremely-low-birth-weight-infants. $J$ Pediatr, 142:656-61.

Ambalavanan N, Kennedy K, Tyson J, et al. 2004. Survey of vitamin A supplementation for extremely-low-birth-weight infants: is clinical practice consistent with the evidence? J Pediatr, 145:304-7.

Bernhard W, Mottaghian J, Gebert A, et al. 2000. Commercial versus native surfactants. Surface activity, molecular components, and the effect of calcium. Am J Respir Crit Care Med, 162:1524-33.

Biesalski HK, Reifen R, Furst RP, et al. 1999. Retinyl palmitate supplementation by inhalation of an aerosol improves vitamin A status of preschool children in Gondar (Ethiopia). Br J Nutr, 82:179-82.

Bronstein V, Khashu M, Singh A, et al. 2005. Exploring a novel mode of vitamin A supplementation: intratracheal administration with surfactant [abstract]. Pediatr Res, 57:2507.

Brooks AD, Tong W, Benedetti F, et al. 2000. Inhaled aerosolization of alltrans-retinoic acid for targeted pulmonary delivery. Cancer Chemother Pharmacol, 46:313-18.

D’Angio CT, Maniscalco WM. 2004. Bronchopulmonary dysplasia in preterm infants: pathophysiology and management strategies. Pediatric Drugs, 6:303-30.

Dahl AR, Grossi IM, Houchens DP, et al. 2000. Inhaled isotretinoin (13-cis retinoic acid) is an effective lung cancer chemopreventive agent in A/J mice at low doses: a pilot study. Clin Cancer Res, 6:3015-24.

Darlow BA, Graham PJ. 2002. Vitamin A supplementation for preventing morbidity and mortality in very low birthweight infants. Cochrane Database Syst Rev, 4:CD000501.

Eggert LD, Rusho WJ, MacKay MW, et al. 1982. Calcium and phosphorus compatibility in parenteral nutrition solutions for neonates. Am J Hosp Pharm, 39:49-53.

Enhorning G. 1977. Pulsating bubble technique for evaluating pulmonary surfactant. J Appl Physiol, 43:198-203.

Enhorning G. 2001. Pulmonary surfactant function studied with the pulsating bubble surfactometer (PBS) and the capillary surfactometer (CS). Comp Biochem Biophysiol, 129:221-6.

Francisko A, Chakaraborty J, Yunus M, et al. 1993. Acute toxicity of vitamin A given with vaccine in infancy. Lancet, 342:526-7.

Haas C, Genzel-Boroviczeny O, Koletzko B. 2002. Losses of vitamin A and $\mathrm{E}$ in parenteral nutrition suitable for premature infants. Eur J Clin Nutr, 56:906-12.

Hustead VA, Gutcher GR, Anderson SA, et al. 1984. Relationship of vitamin A (retinol) status to lung disease in the preterm infant. J Pediatr, 105:610-15.

Kohlhaufl M, Haussinger K, Stanzel F, et al. 2002. Inhalation of aerosolized vitamin A: reversibility of metaplasia and dysplasia of human respiratory epithelia-a prospective pilot study. Eur $J$ Med Res, $7: 72-8$.

Lavoie JC, Chessex P, Rouleau T, et al. 2007. Shielding parenteral multivitamins from light increases vitramin $\mathrm{A}$ and $\mathrm{E}$ concentration in lung of newborn guinea pigs. Clinical Nutr, 26:341-7.

Maden M, Hind M. 2004. Retionoic acid in alveolar development, maintenance and regeneration. Phil Trans R Soc Lond, B359:799-808. 
McMillan D, Chernick V, Finer N, et al. 1995. Effects of two rescue doses of synthetic surfactant in 344 infants with respiratory distress syndrome weighing 750 to 1249 grams: a double-blind, placebo-controlled multicenter Canadian trial. Canadian Exosurf Neonatal Study Group. J Pediatr, 126:S90-8.

Ozer EA, Kumral A, Ozer E, et al. 2005. Effect of retinoic acid on oxygen induced lung injury in the newborn rat. Pediatr Pulmonol, 39:35-40.

Shenai JP, Chytil F, Stahlman MT. 1985. Vitamin A status of neonates with bronchopulmonary dysplasia. Pediatr Res, 19:185-8.

Smith VC, Zupancic JA, MvCormick MC, et al. 2004. Rehospitalisation in the first year of life among infants with bronchopulmonary dysplasia. J Pediatr, 144:799-803.

Snyder JM, Jenkins-Moore M, Jackson SK, et al. 2005. Alveolarization in retinoic acid receptor-beta-deficient mice. Pediatr Res, 57:384-91.

Stenmark KR, Abman SH. 2005. Lung vascular development: implication for the pathogenesis of bronchopulmonary dysplasia. Ann Rev Physiol, 67:623-61.
Tyson JE, Wright LL, Oh W, et al. 1999. Vitamin A supplementation for extremely-low-birthweight infants: National Institute of Child Health and Human Development Neonatal Research Network. N Engl J Med, 340:1962-8.

Wardle SP, Hughes A, Chen S, et al. 2001. Randomized controlled trial of oral vitamin A supplementation in preterm infants to prevent chronic lung diseases. Arch Dis Child Fetl Neonatal Ed, 84:F10-13.

Wong JC, McDougal AR, Tofan M, et al. 2006. Doubling calcium and phosphate concentrations in neonatal parenteral nutrition solutions using monobasic potassium phosphate. J Am Coll Nutr, 25:70-7. 